\title{
Video Article \\ Small-scale Nuclear Extracts for Functional Assays of Gene-expression Machineries
}

\author{
Eric G. Folco ${ }^{1}$, Haixin Lei ${ }^{1}$, Jeanne L. Hsu ${ }^{1}$, Robin Reed ${ }^{1}$ \\ ${ }^{1}$ Department of Cell Biology, Harvard Medical School \\ Correspondence to: Robin Reed at Robin_Reed@hms.harvard.edu \\ URL: https://www.jove.com/video/4140 \\ DOI: doi:10.3791/4140
}

Keywords: Cellular Biology, Issue 64, Genetics, HeLa nuclear extract, small-scale extract, pre-mRNA splicing, RNA polymerase II transcription, $\mathrm{RNAi}$, coupled transcription/splicing, in vitro gene expression assays

Date Published: 6/27/2012

Citation: Folco, E.G., Lei, H., Hsu, J.L., Reed, R. Small-scale Nuclear Extracts for Functional Assays of Gene-expression Machineries. J. Vis. Exp. (64), e4140, doi:10.3791/4140 (2012).

\section{Abstract}

A great deal of progress in understanding gene expression has been made using in vitro systems. For most studies, functional assays are carried out using extracts that are prepared in bulk from 10-50 or more liters of cells grown in suspension. However, these large-scale preparations are not amenable to rapidly testing in vitro effects that result from a variety of in vivo cellular treatments or conditions. This journal video article shows a method for preparing functional small-scale nuclear extracts, using HeLa cells as an example. This method is carried out using as few as three $150 \mathrm{~mm}$ plates of cells grown as adherent monolayers. To illustrate the efficiency of the small-scale extracts, we show that they are as active as bulk nuclear extracts for coupled RNA Polymerase II transcription/splicing reactions. To demonstrate the utility of the extract protocol, we show that splicing is abolished in extracts prepared from HeLa cells treated with the splicing inhibitor drug E7107. The smallscale protocol should be generally applicable to any process or cell type that can be investigated in vitro using cellular extracts. These include patient cells that are only available in limited quantities or cells exposed to numerous agents such as drugs, DNA damaging agents, RNAi, or transfection, which require the use of small cell populations. In addition, small amounts of freshly grown cells are convenient and/or required for some applications.

\section{Video Link}

The video component of this article can be found at https://www.jove.com/video/4140/

\section{Protocol}

\section{Growing HeLa Cells for Nuclear Extraction}

1. Plate HeLa cells on three $150 \mathrm{~mm}$ plates with DMEM media supplemented with $10 \% \mathrm{FBS}$ and $1 \%$ Penicillin/Streptomycin. Grow in a $37{ }^{\circ} \mathrm{C}$ incubator with $5 \% \mathrm{CO}_{2}$ until cells are $90 \%$ confluent.

Tip- Split cells 1:10 from a confluent plate and harvest 3 days after splitting.

Tip- An extra plate can be grown in case there are not enough cells with three plates (for example, if the cells are less than $90 \%$ confluent).

\section{Prepare and Chill Nuclear Extract Solutions}

1. Prepare fresh solutions as outlined in Table 2.

2. Aliquot $10 \mathrm{ml}$ of Hypotonic Buffer into a $15 \mathrm{ml}$ Falcon tube. Aliquot $1 \mathrm{ml}$ of High Salt Buffer and $1 \mathrm{ml}$ of Low Salt Buffer into $1.5 \mathrm{ml}$ Eppendorf tubes. Chill the buffers on ice. Add the appropriate volumes of PMSF and DTT to each buffer immediately prior to use (see Table 1).

\section{Harvesting HeLa Cells for Nuclear Extraction}

1. Aspirate media from the cells and wash the cells once with $13 \mathrm{ml}$ of room temperature $1 \mathrm{X}$ PBS per plate.

2. Aspirate the PBS wash.

Tip- Aspirate as much of the PBS as possible by first aspirating, then standing the plate on its side, waiting a few seconds, and aspirating the rest.

3. Using a cell lifter, scrape cells to the bottom edge of the plates, and then transfer the cells to an Eppendorf tube. Avoid making bubbles. The 3 plates of scraped cells should fit into one $1.5 \mathrm{ml}$ Eppendorf tube. 
Tip- It is easier to estimate the volume of cells if an Eppendorf tube is used. For scale up, use Falcon tubes.

4. Centrifuge at $4{ }^{\circ} \mathrm{C}$ in a microfuge for $5 \mathrm{~min}$ at $100 \mathrm{xg}$ to pellet the cells.

\section{Swell HeLa Cells in Hypotonic Buffer}

1. Carefully aspirate the PBS from the cell pellet and estimate the Packed Cell Volume (PCV) by looking at the gradation on the tube. Three confluent plates of HeLa cells yield about $500 \mu$ l of PCV.

2. Add $2 X$ the PCV of Hypotonic Buffer to the cell pellet. Shake gently to resuspend the cells. Use a P1000 Pipetman to gently resuspend the cells by pipetting up and down if necessary.

3. Centrifuge at $4{ }^{\circ} \mathrm{C}$ in a microfuge for 5 min at $100 \times \mathrm{g}$ to pellet the cells. ${ }^{*}$ The cells will have begun to swell by this time, and the cell volume should now be about $750 \mu \mathrm{l}-1000 \mu \mathrm{l}$.

4. Carefully aspirate the Hypotonic Buffer and add new Hypotonic Buffer to a final volume of $1.5 \mathrm{ml}$. Resuspend the cells.

5. Incubate the resuspended swollen cells on ice for $10 \mathrm{~min}$.

\section{Lyse Cells Using a Dounce Homogenizer}

1. Use Hypotonic Buffer to rinse the dounce and then chill it on ice. Wipe the pestle dry with a delicate task wiper (e.g. Kimwipe). Remove the remaining buffer from the dounce using a $1000 \mu$ lextended micropipette tip.

2. Transfer swollen cells into the dounce. Slowly move the tight pestle of the dounce up and down 5 times to lyse the cells, being careful to avoid bubbles.

3. Assay for cell lysis using a $5 \mu \mathrm{l}$ aliquot of cells from the dounce. Place the aliquot in an Eppendorf tube and add an equal volume of Trypan Blue. Put the mixture on a slide and examine the cells using a light microscope. The nuclei of lysed cells will appear blue. About $90 \%$ lysis is ideal.

Tip- The number of times the cells should be dounced will vary depending upon the tightness of the dounce. When carrying out this protocol for the first time, determine the number of times to dounce by carrying out step 5.3 after each stroke with the dounce. Do not over-dounce, excessive douncing will destroy the nuclei.

4. Transfer the lysed cells to a pre-chilled Eppendorf tube and spin in a $4{ }^{\circ} \mathrm{C}$ microfuge for $5 \mathrm{~min}$ at $1500 \mathrm{x}$. The pellet contains the nuclei. Carefully transfer the supernatant to a new tube without disrupting the nuclei. The supernatant contains the cytoplasm.

Tip- The cytoplasm can be used as is or further processed to an $\mathrm{S} 100$ by using the same high-speed spin used for bulk extracts (see ${ }^{1}$ for preparing an active S100 from bulk extracts).

\section{Salt-Extract the Nuclei}

1. Estimate the Packed Nuclear Volume (PNV) by looking at the gradation on the tube. A PCV of $500 \mu l$ typically yields a PNV of $400 \mu \mathrm{l}$.

2. Add $1 / 2 X$ PNV of Low Salt Buffer to the nuclei by inserting a micropipette tip to the bottom of the tube and slowly squirting the buffer into the nuclei while gently mixing. Do not pipet up and down. Gently flick the tubes to make sure that the pellet is completely resuspended in the Low Salt Buffer before continuing.

3. Add $1 / 2 \times$ PNV of High Salt Buffer and quickly mix 1 time by inverting the tube. Do not shake. Rotate at $4{ }^{\circ} \mathrm{C}$ for $30 \mathrm{~min}$.

Tip- Be gentle with the nuclei when they are in High Salt Buffer to avoid lysing them.

4. Spin at $4{ }^{\circ} \mathrm{C}$ in a microfuge for $15 \mathrm{~min}$ at $18,000 \mathrm{xg}$. The supernatant is the high salt nuclear extract (HS-NE). Three plates of cells typically yield $500-600 \mu \mathrm{l}$ of HS-NE.

\section{Concentrate and Dialyze the Nuclear Extract}

1. Transfer $500 \mu \mathrm{l}$ of the HS-NE into chilled mini-centricons (Amicon) and spin for $50 \mathrm{~min}$ at $4{ }^{\circ} \mathrm{C}$ in a microfuge at $14000 \times \mathrm{g}$. Invert the mini-centricon (Amicon) and place it in a new Eppendorf tube. Spin for $2 \mathrm{~min}$ at $4{ }^{\circ} \mathrm{C} 1000 \mathrm{xg}$ to recover the HS-NE. The HS-NE will be concentrated to approximately $115 \mu \mathrm{l}$.

2. Using a P200 Pipetman, transfer $45 \mu$ aliquots of the HS-NE into mini-dialysis Slide-A-Lyzers, and place them in $500 \mathrm{ml}$ of chilled Dialysis Buffer. Ensure that the bottom of the Slide-A-Lyzer is aligned with the bottom of the floater. Stir for $1-2$ hrs at $4{ }^{\circ} \mathrm{C}$. The NE will appear cloudy after dialysis.

Tip- Do not centrifuge to remove the cloudy precipitate that is observed after dialysis, as this centrifugation reduces the activity of the extract.

3. The NE can be used immediately or aliquoted. Aliquots should be flash frozen in liquid nitrogen and stored at $-80{ }^{\circ} \mathrm{C}$. The $\mathrm{NE}$ can be stored and undergo at least two freeze-thaw cycles without losing activity.

\section{Representative Results}

Recently, efficient in vitro systems for coupling RNAP II transcription to splicing were developed ${ }^{2-5}$. These systems employed HeLa cells grown in bulk and thus are not amenable to rapidly testing the effects of specific cellular treatments on multiple samples. Based on this need and the general utility of a small-scale extract protocol (see Discussion), we established a robust small-scale nuclear extract method. Representative data comparing the RNAP II transcription/splicing reaction in the small-scale nuclear extract with the bulk nuclear extract is shown in Figure 
2. A CMV-DNA construct, which contains the CMV promoter and encodes a standard splicing substrate (Ftz ${ }^{3}$, Figure 2A) was used for the analysis. When this construct was incubated in the bulk (lanes 1-3) or small-scale (lanes 4-6) extract, similar levels of the nascent pre-mRNA were synthesized by the 5 min time point (Figure 2, lanes 1 and 4). Following addition of $\alpha$-amanitin to block further transcription, the splicing intermediates and spliced products accumulated over time with similar kinetics in both types of extracts (Figure 2, lanes 2, 3, 5, 6). These representative results show that the efficiency of the coupled RNAP II transcription/splicing system is similar in the bulk and small-scale nuclear extracts.

To demonstrate the utility of the small-scale extract method, extracts were prepared from HeLa cells treated with the splicing inhibitor, E7107, or the negative control compound pladienolide $\mathrm{F}^{6,7}$ and then the coupled RNAP II transcription/splicing assay was carried out. As shown in Figure 3, transcription by RNAP II occurred efficiently in extracts prepared from both the Pladienolide F and E7107 treated cells (10 min time points). In contrast, splicing occurred normally in the extract prepared from the Pladienolide F-treated cells but was abolished in the E7107-treated cells (Figure 3, 20-60 min time points). These data provide proof of concept for using the small-scale nuclear extracts for special treatment of cells in small scale.

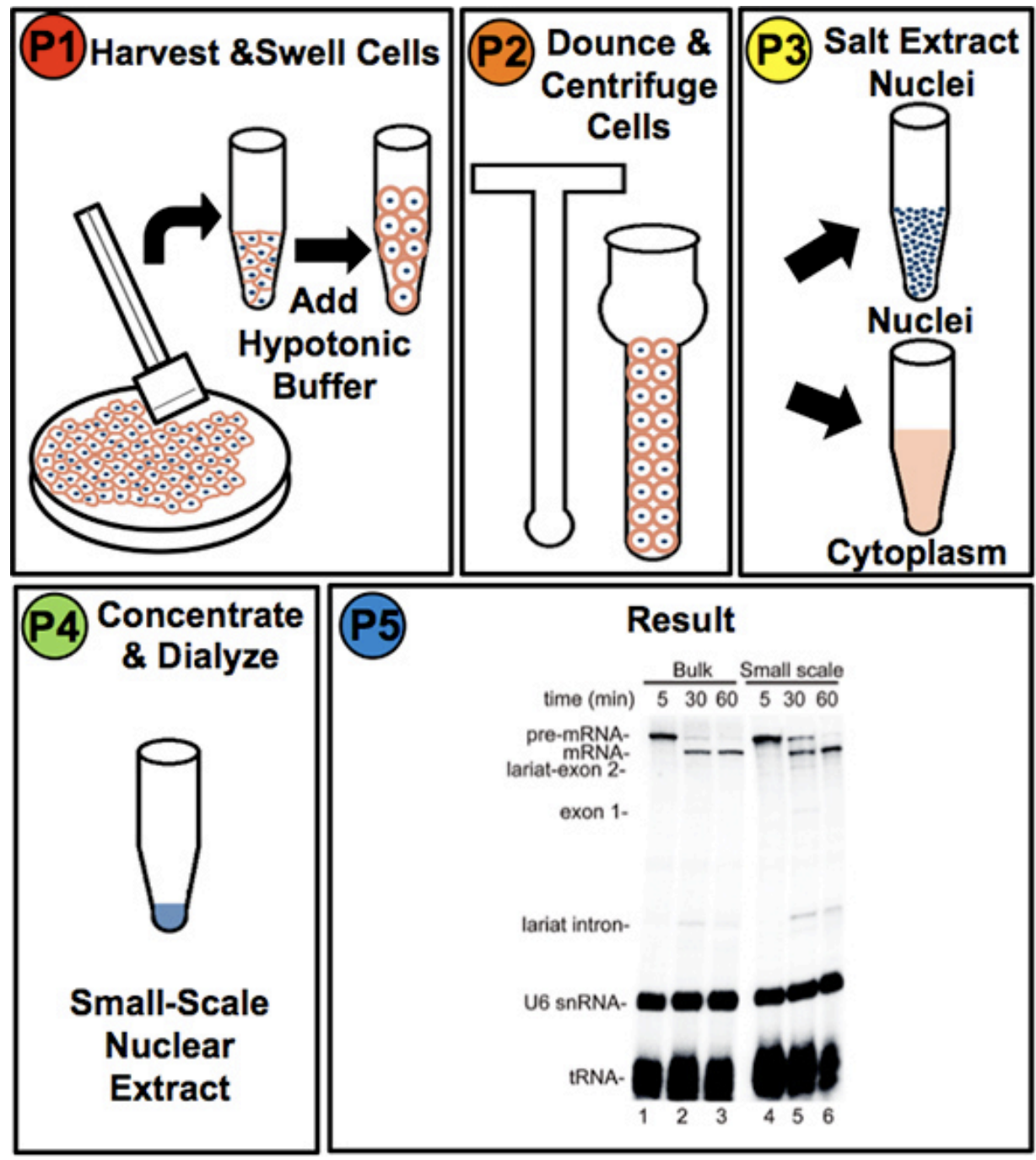

Figure 1. Schematic of Small-scale Extract Protocol. Step P1. Cells are grown as monolayers, harvested from plates using a cell lifter and swollen by the addition of hypotonic buffer. Step P2. Cells are lysed using a Dounce homogenizer and centrifuged to pellet the nuclei. Step P3. Nuclei are separated from the cytoplasm and undergo salt extraction. Step P4. The nuclear extract is concentrated and dialyzed. Step P5. Results are obtained that show that nuclear extracts are functional (see Figure $\mathbf{2}$ for greater detail). 


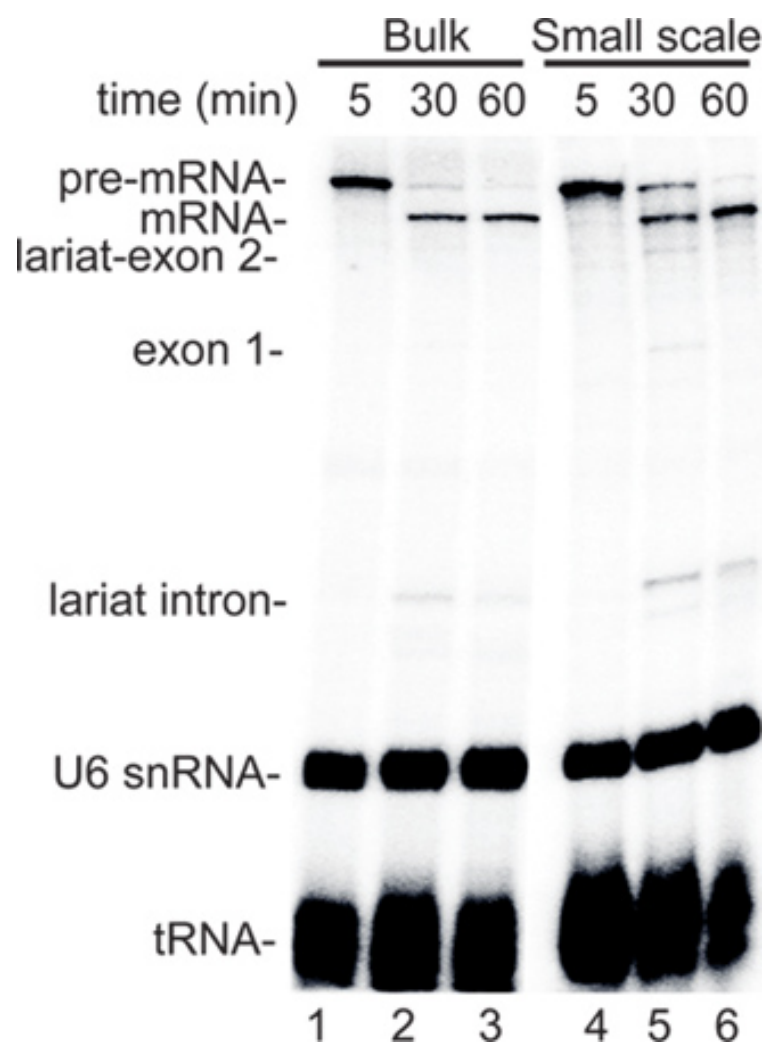

Figure 2. Small-scale nuclear extracts are robust in a coupled RNAP II transcription/splicing assay. A. Schematic of the CMV-Ftz DNA template used for coupled RNAP II transcription/splicing. The CMV promoter and the sizes of the exons and intron are indicated. B. Comparison of coupled RNAP II transcription/splicing assay using either bulk nuclear extract or small-scale nuclear extract. $\alpha$-amanitin was added after 5 min of transcription and splicing was allowed to occur for 30 and 60 min. RNA was extracted and fractionated on a $5 \%$ denaturing polyacrylamide gel and detected by phosphoimager. The splicing intermediates and products are indicated. The endogenous U6 snRNA and tRNA present in the extract and that are ${ }^{32} \mathrm{P}$-labeled during incubation are indicated. See ${ }^{3}$ for a detailed protocol on the coupled RNAP II transcription/splicing system. 


\section{Pladienolide $\mathrm{F}$ \\ time (min) $10 \quad 20 \quad 30 \quad 45 \quad 60 \quad 10 \quad 20 \quad 30 \quad 45 \quad 60$

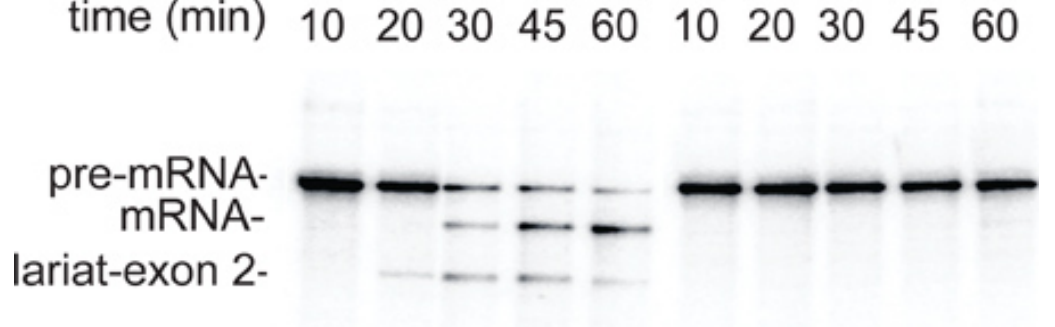

exon 1-

\section{lariat-intron-}

\section{U6 snRNA- \\ tRNA-

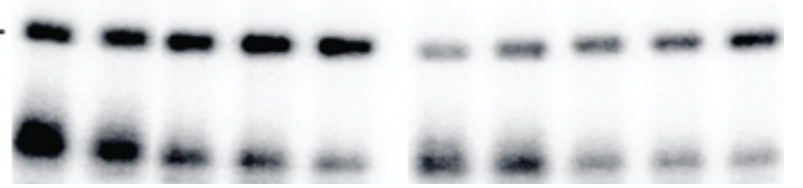

Figure 3. Small-scale nuclear extracts prepared from HeLa cells treated with the splicing inhibitor drug E7107 are defective in splicing. Cells were treated with $3 \mu \mathrm{M}$ Pladienolide F or E7107 as described ${ }^{6}$ and then used to prepare small-scale nuclear extracts. A time course was carried out using the same transcription/splicing assay as in Figure 2. The splicing intermediates and products, and U6 snRNA and tRNA are indicated.

\section{Discussion}

We have established a rapid and reproducible method for preparation of nuclear extracts from small quantities of HeLa cells grown as monolayers. We demonstrated that these extracts are robust by showing that the kinetics and efficiency of RNAP II transcription/splicing assays are similar in the small-scale and bulk nuclear extracts. We showed the utility of the extracts by demonstrating that extracts prepared from cells treated with a splicing inhibitor drug are active for transcription but defective in splicing.

Our method for preparing small-scale nuclear extracts was established by combining and optimizing methods previously established for making extracts from HeLa cells grown in suspension in large-scale ${ }^{1,8}$ and a method for small-scale preparation of extracts ${ }^{9}$. We established our protocol because the previous small-scale extracts were not functional for some assays, such as the coupled transcription/splicing assay. An important difference between the previous small-scale protocol ${ }^{9}$ and ours is that we optimized conditions for lysing cells using a mini-dounce whereas the previous protocol lysed cells by pushing them through a small-gauge needle ${ }^{9}$. The needle-lysis results in bubbles, which may explain why the extracts were inactive and/or difficult to reproduce for some assays. We also added a concentration step to our protocol. This step increases the activity of the extracts, which are extremely sensitive to concentration, and also limits the variability between extracts that is inherent to smallscale preparations. Finally, our preparation is routinely carried out with only three $150 \mathrm{~mm}$ plates of monolayer cells, without any significant effect on activity compared to bulk extracts. Thus, the procedure is readily amenable for small-scale preparations that require costly reagents or limited cell availability. For example, we have prepared small-scale extracts from RNAi knockdown cells and from Chronic Lymphocytic Leukemia (CLL) patient cells, and used these extracts for functional and/or biochemical assays (EGF, JLH, TY and RR, unpublished). We have found that the extracts are valuable for RNAi followed by specific biochemical assays, such as immunopreciptations, Westerns, and silver staining. After establishing the efficacy of knocking down a particular protein, a more detailed study can be carried out by making a stable knockdown cell-line, which can be used to prepare additional extracts at lower cost. Mass spectrometry of proteins present in immunoprecipitates obtained from these knockdown cell lines will also be a useful application of the method. In addition to the coupled transcription/splicing assay that we used as an example for our protocol description here, the small-scale extracts should be generally applicable to numerous functional and biochemical assays, such as those used for the different steps in gene expression (e.g. capping, splicing, transcription, polyadenylation, microRNA processing). The protocol can also be adapted for patient cell types that can be either obtained in suspension (such as the CLL cells) or grown in culture (such as patient fibroblasts). Finally, the cytoplasmic fraction obtained during the procedure should prove useful for both functional and biochemical assays that require the cytoplasm.

\section{Disclosures}

Production and Free Access to this article is sponsored by Abcam, Plc. 


\section{Acknowledgements}

We are grateful to M. Winkelbauer-Hurt, E. Ibrahim, P. Valencia, K. Dufu, H. Cheng for useful discussions. HeLa cells were obtained from the National Cell Culture Center (Minneapolis, MN). We also thank Eisai Co., Ltd for providing E7107 and the Nikon Imaging Center at Harvard Medical School for help with light microscopy. This work was supported by an NIH grant GM043375 to RR and EGF and NRSA fellowship to JLH.

\section{References}

1. Krainer, A.R., Maniatis, T., Ruskin, B., \& Green, M.R. Normal and mutant human beta-globin pre-mRNAs are faithfully and efficiently spliced in vitro. Cell. 36, 993-1005 (1984).

2. Ghosh, S. \& Garcia-Blanco, M.A. Coupled in vitro synthesis and splicing of RNA polymerase II transcripts. Rna. 6, 1325-1334 (2000).

3. Das, R., et al. Functional coupling of RNAP II transcription to spliceosome assembly. Genes Dev. 20, 1100-1109 (2006).

4. Hicks, M.J., Yang, C.R., Kotlajich, M.V., \& Hertel, K.J. Linking splicing to Pol II transcription stabilizes pre-mRNAs and influences splicing patterns. PLoS Biol. 4, e147 (2006).

5. Yu, Y., Das, R., Folco, E.G., \& Reed, R. A model in vitro system for co-transcriptional splicing. Nucleic Acids Res. 38, 7570-7578, doi:10.1093/nar/gkq620 [pii] gkq620 (2010).

6. Kotake, Y., et al. Splicing factor SF3b as a target of the antitumor natural product pladienolide. Nat. Chem Biol. 3, 570-575, doi:10.1038/ nchembio.2007.16 [pii] nchembio.2007.16 (2007).

7. Folco, E.G., Coil, K.E., \& Reed, R. The anti-tumor drug E7107 reveals an essential role for SF3b in remodeling U2 snRNP to expose the branch point-binding region. Genes \& development. 25, 440-444, doi:10.1101/gad.2009411 (2011).

8. Dignam, J.D., Lebovitz, R.M., \& Roeder, R.G. Accurate transcription initiation by RNA polymerase II in a soluble extract from isolated mammalian nuclei. Nucleic Acids Res. 11, 1475-1489 (1983).

9. Lee, K.A., Bindereif, A., \& Green, M.R. A small-scale procedure for preparation of nuclear extracts that support efficient transcription and premRNA splicing. Gene Anal. Tech. 5, 22-31 (1988). 